\title{
The European Academy of Paediatric Dentistry and Molar Incisor Hypomineralisation
}

\author{
K. Weerheijm ${ }^{1}$
}

Published online: 7 May 2015

(C) European Academy of Paediatric Dentistry 2015

In September 2003, the European Academy of Paediatric Dentistry (EAPD) published its first special issue (Volume 4 issue 3, 2003). The general theme of this special issue was Molar Incisor Hypomineralisation (MIH). Besides prevalence figures, (restorative) treatment, fluoride intake and MIH, a description of the phenomenon of MIH and EAPD judgement criteria for MIH in epidemiologic studies were proposed.

The EAPD has been a pioneer in distributing and sharing MIH knowledge and research-related matters with their members. In the December 2008 issue (Volume 9 issue 4, 2008), an MIH update was given as the basis for the upcoming 2009 EAPD MIH Interim Symposium in Helsinki, Finland. The results of this Helsinki meeting discussions can be found in the April issue of 2010 (Volume 11 issue 2, 2010).

At the moment, it is 2015; about 1 year after the MIH Workshop held in Sopot Poland at the 12th EAPD congress and 12 years after the first special issue. The time has come for an update and a second MIH special issue.

As Martin Curzon stated in his 2003 editorial, already in the 1940s the best time for extraction of the first permanent molars was discussed. Nowadays, despite the decline in caries prevalence, the first permanent molar extraction is still a serious treatment option. At that time, the high caries prevalence was the main reason for the extraction of the 'keystone' molars. Today, enamel disturbances such as MIH contribute to this.

Although early diagnosis of MIH with preventive follow-up immediately after eruption is the most important

K. Weerheijm

weerkamp@xs4all.nl

1 Paediatric Research Project (PREP), Amsterdam, The Netherlands approach in the treatment of such molars, sometimes the defects are too severe to restore the molars in the long term. This brings us back to the 1940s discussions when to extract this important molar.

In 2015, the aetiology of MIH is still unknown but more knowledge has been gained about the clinical characteristics of the opacities. As far as we know now, the aetiology seems to be multifactorial with maybe a genetic predisposition. Evidence of specific factors remains weak, resulting in a lack of consensus regarding protocols and limited understanding regarding the exact pathogenesis.

Research has also been focused on primary molars. This resulted in reports of comparable lesions in hypomineralised second primary molars (HSPM). The presence of HSPM and demarcated opacities in erupting permanent incisors can be used as one of the risk indicators of MIH. Also colour and location of the demarcated opacities in MIH-affected molars are indicative of the relative risk for post-eruptive enamel breakdown: clinician's tools for an early diagnosis and helpful in proper treatment planning.

Although the EAPD criteria have been used extensively since 2003, prevalence ratings of MIH found in epidemiological surveys still differ considerably and are sometimes difficult to interpret. Representative data of countries are missing. Most values mentioned are only representative for specific areas. There is a need for a worldwide-accepted standardised scoring and calibration system. Standardisations of surveys contain also a minimum number of participants (at least 300 for prevalenceand 1000 for prospective aetiology studies) per study.

During examination of children as a part of MIH or HSPM studies, we always have to keep in mind the purpose of the study and the original reason for the definition of MIH.

In MIH research, we have to distinguish between clinical and research relevance. We all know that opacities- 
also demarcated ones-can be found throughout the whole dentition. We also know that not all opacities have the same origin. While even demarcated opacities and other enamel defects can look identical to MIH. Especially in daily dental practice, the clinical pattern of recognising first permanent molars with or without incisor involvement is important. As researchers we should never forget the clinical relevance and importance of our research. This means that in MIH research, sometimes a simple screening study with a short form with only target teeth will do, for example, MIH and HSPM prevalence studies as part of epidemiological caries prevalence studies. On the other hand, if we want to get deeper into the problem with longitudinal observational research, a longer form could be more appropriate.

In Sopot, it became clear that maybe it is time to update the EAPD 2003 and 2009 criteria once again. A review of MIH prevalence data until the end of 2014 makes it clear that standardisation of the MIH research is still one of the first priorities. A suggestion for a new updated scoring system with a long- and a short-form variant with photographs for calibration is the opening article of this issue. Due to the generosity of sponsors, we are able to provide this article to you as an open access article.

The other MIH-related manuscripts in this special issue-I hope-would also trigger our research genes. With new insight of the influence of fluoride on MIH opacities, prevalence of only opacities on incisors without molar involvement, prevalence figures out of Nigeria and the severity of MIH molars in a German school population. Things to think about and results to discuss with colleagues.

I wish you a lot of reading pleasure and new ideas for MIH and HSPM research, hopefully resulting in a third special issue providing us with answers to the remaining questions concerning this child unfriendly enamel disturbance. 